\title{
Analysis of correlation and ionization from pair distributions in many-electron systems
}

\author{
S. López-Rosa ${ }^{1,2, a}{ }_{\mathbb{D}}$, J. C. Angulo ${ }^{2,3, b}$, A. L. Martín ${ }^{4}$, J. Antolín ${ }^{2}$ \\ ${ }^{1}$ Departamento de Física Aplicada II, Universidad de Sevilla, 41012 Sevilla, Spain \\ 2 Instituto Carlos I de Física Teórica y Computacional, Universidad de Granada, 18071 Granada, Spain \\ 3 Departamento de Física Atómica, Molecular y Nuclear, Universidad de Granada, 18071 Granada, Spain \\ 4 Berlin Institute for Medical Systems Biology, Max Delbrück Center for Molecular Medicine in the Helmholtz \\ Association, 10115 Berlin, Germany
}

Received: 21 January 2021 / Accepted: 10 July 2021

(C) The Author(s) 2021

\begin{abstract}
Jensen-Shannon divergence is used to quantify the discrepancy between the Hartree-Fock pair density and the product of its marginals for different $N$-electron systems, enclosing neutral atoms (with nuclear charge $Z=N$ ) and singly-charged ions $(N=Z \pm 1)$. This divergence measure is applied to determine the interelectronic correlation in atomic systems. A thorough study was carried out, by considering (i) both position and momentum conjugated spaces, and (ii) systems with a nuclear charge as far as $Z=103$. The correlation among electrons was measured by comparing, for an arbitrary system, the double-variable electron-pair density with the product of the respective one-particle densities. A detailed analysis throughout the Periodic Table highlights the relevance not only of weightiness for the systems considered, but also of their shell structure. Besides, comparative computations between two-electron densities of different atomic systems (neutrals, cations, anions) quantify their dissimilarities, patently governed by shell-filling patterns throughout the Periodic Table.
\end{abstract}

\section{Introduction}

The two-electron density $\Gamma\left(\mathbf{r}_{1}, \mathbf{r}_{2}\right)$ is the probability density of any two electrons that are located at radii $r_{1}$ and $r_{2}$, respectively, and is a convenient starting point to study the spatial interaction between two electrons in an explicit manner [1]. This density provides a quantummechanical description of the distribution of electron pairs in the space and therefore represents a central tool, in quantum chemistry, to study chemical binding [2] or electronic structure and correlation in molecular systems $[3,4]$.

On the other hand, the exact interaction energy of a many-electron system is determined by the electron pair density, but is not well-approximated in standard Kohn-Sham density functional models [5,6]. Consequently, pair density functional theory is regarded as not only an extension of the density functional theory but a reduced density matrix theory as well $[7,8]$. The main feature of the pair density functional theory is that the pair density, as a basic

\footnotetext{
a e-mail: slopezrosa@us.es (corresponding author)

be-mail: angulo@ugr.es
} 
variable, essentially contains more information on the electron-electron interaction than the electron density $[9,10]$.

The expectation value of an arbitrary two-particle operator can be obtained using only the electron pair density. In addition to this merit, the pair density functional theory has another, which is related to the expansion of the approximate functional. That is to say, in the pair density functional theory, the only required selection is the approximate form of the kinetic energy functional, because the exchange-correlation energy functional can rigorously be expressed by means of the pair density [11]. As a recent example multiconfiguration pair density functional theory has been developed, as a way to combine the advantages of both wave function and density functional theories to provide a better treatment of strongly correlated systems, and its results have been compared to those of some Kohn-Sham density functionals, including both traditional and modern functionals [12,13]. Additional applications have been performed recently with a diversity of molecular systems, on the basis of their one- and two-electron RDMs (reduced density matrices), which comparison provides measures of electron-correlation and entanglement among systems. These measures display their sensitivity to the presence of electric fields [14].

Therefore, given the above, it would be logical to assume that two-electron densities for atoms and molecules have been studied both intensively and extensively. However, this is not the case. The effort invested in interpretative studies of electron pair densities has been small as compared to that expended on such studies for one-electron densities. Indeed, there is a large amount of information coded inside monoelectronic densities; however, these densities do not give us any hint on how the position (or the momentum) of an electron conditions the positions (or the momenta) of the others. Besides it is worthy to note that within the WallerHartree theory, the two-electron density is related to the total X-ray scattering intensity which is accessible by experimental measurements $[15,16]$.

One of the main difficulties rests in the interpretation of the electron pair density. Visual representation is a powerful analytical tool; however, being a function of 6 coordinates, graphical representation of the pair density is not feasible and manipulations to the twoelectron density must be carried out to extract useful information. One such manipulation gives place to the intracule and extracule densities and many relevant results have been obtained in this framework [17-21]. It is important to note that these densities consider the pairs of electrons as a whole thing. Other simplification consists in dealing with spherically averaged electron pair densities. Consequently, as a result of this reduction in dimensionality, some two-electron information is lost in the transformation.

The concepts of uncertainty, randomness, disorder and delocalization are basic ingredients in the study, within an information-theoretic framework, of relevant structural properties for many different probability distributions appearing as descriptors of several chemical and physical systems and processes. The relevancy of these concepts has motivated new studies that pursue quantification, giving rise to a variety of density functionals, such as Shannon entropy [22], Fisher information [23], disequilibrium [24], complexity [25], and many others. These information measures have been widely employed for describing the information content and behavior in a great variety of fields [26,27] and, in particular, for the study of many-electron systems [27-34]

Following the usual procedures carried out within information theory for quantifying the uncertainty or disorder of individual distributions, some extensions have been made in order to introduce the concepts of 'distance' and 'divergence' between two distributions, as comparative measures of their dissimilarity.

Jensen-Shannon divergence (JSD) is a powerful divergence measure that represents the difference between the Shannon entropy of the mean density and the mean value of the indi- 
vidual entropies [35-37]. Its non-negativity arises from the convex character of the Shannon entropy functional. This comparative measure has found deep applications in statistics and many other fields, including entanglement characterization [38], analysis of symbolic sequences or series, and in particular in the study of segmentation of DNA sequences [39]. JSD and some effective generalizations have been used for the study and comparison of one-electron densities [40-44]. For the first time, to the best of our knowledge, this information measure of dissimilarity is applied to the study of two-electron density functions. Let us emphasize, however, recent applications of strongly related functionals (e.g. mutual information, cumulative residual entropies, interaction information) for uncoupled and interacting harmonic oscillators [45,46]. Additional applications include the cumulant part of two-electron RDMs, for the detection of van der Waals interactions between specific parts of molecular systems [47]. Other measures enclosing also the gradient of the density, as the relative Fisher information, were considered for the study of specific one-particle quantum systems (Morse potential, isotropic oscillator or hydrogen-like atoms) $[48,49]$.

In this paper, small correlation effects in atoms are analyzed, on the basis of discrepancies between the Hartree-Fock one- and two-electron densities. In doing so, comparative density functionals are employed as quantifiers of dissimilarity among the above densities. So differences arising from the exchange term are emphasized, as the main cause of them. A detailed analysis throughout the Periodic Table reveals the relevance not only of weightiness for the systems considered, but also of their shell structure. Besides, comparative computations between two-electron densities of different atomic systems (neutrals, cations, anions) quantify the important differences patently governed by shell-filling patterns throughout the Periodic Table. Thereby, we use an information-theoretic functional that has shown its universality and versatility in other computations: the Jensen-Shannon divergence (JSD) [35-37].

The organization of the paper is the following: in the next section, two-particle densities are defined and presented for atomic systems and also the entropic divergence measure (JSD) we are going to use for comparing them. Numerical computations and results are presented in Sect. 3. Finally, conclusions and open problems or future work are briefly described in the last section.

\section{Electron pair densities and related measures}

In terms of the $N$-electron wave function $\Psi$, and its Fourier transform $\Phi$, the two-electron densities are defined as

$$
\Gamma\left(\mathbf{r}_{1}, \mathbf{r}_{2}\right)=\int \Psi\left(\mathbf{x}_{1}, \mathbf{x}_{2}, \ldots, \mathbf{x}_{N}\right) \Psi^{*}\left(\mathbf{x}_{1}, \mathbf{x}_{2}, \ldots, \mathbf{x}_{N}\right) \mathrm{d} \sigma_{1} \mathrm{~d} \sigma_{2} \mathrm{~d} \mathbf{x}_{3} \ldots \mathrm{d} \mathbf{x}_{N}
$$

in the position space, and

$$
\Pi\left(\mathbf{p}_{1}, \mathbf{p}_{2}\right)=\int \Phi\left(\mathbf{y}_{1}, \mathbf{y}_{2}, \ldots, \mathbf{y}_{N}\right) \Phi^{*}\left(\mathbf{y}_{1}, \mathbf{y}_{2}, \ldots, \mathbf{y}_{N}\right) \mathrm{d} \sigma_{1} \mathrm{~d} \sigma_{2} \mathrm{~d} \mathbf{y}_{3} \ldots \mathrm{d} \mathbf{y}_{N}
$$

in the momentum space. The variables $\mathbf{x}_{i}=\mathbf{r}_{i} \sigma_{i}$ and $\mathbf{y}_{i}=\mathbf{p}_{i} \sigma_{i}$ are combined coordinates which include the spin. It is well known that the physical meaning of these densities regards the probability of finding an electron with a compatible state within the region $\mathbf{r}_{1} d \mathbf{r}_{1}$ if there is another electron with allowed/compatible quantum numbers within the region $\mathbf{r}_{2} d \mathbf{r}_{2}$, and similarly regarding the momentum regions $\mathbf{p}_{1} d \mathbf{p}_{1}$ and $\mathbf{p}_{2} d \mathbf{p}_{2}$. These densities are directly related to the electron correlations, as the compatibility of an electron state is in organically 
determined by the compatibility of its state with those of the others. They naturally give us quantifiers of correlation between electrons.

The two-electron densities are going to be calculated by the Hartree-Fock approach and can be expressed as follows [50]:

$$
\Gamma\left(\mathbf{r}_{1}, \mathbf{r}_{2}\right)=\frac{1}{N-1}\left[N \rho\left(\mathbf{r}_{1}\right) \rho\left(\mathbf{r}_{2}\right)-\Gamma_{x}\left(\mathbf{r}_{1}, \mathbf{r}_{2}\right)\right]
$$

in the position space, and

$$
\Pi\left(\mathbf{p}_{1}, \mathbf{p}_{2}\right)=\frac{1}{N-1}\left[N \gamma\left(\mathbf{p}_{1}\right) \gamma\left(\mathbf{p}_{2}\right)-\Pi_{x}\left(\mathbf{p}_{1}, \mathbf{p}_{2}\right)\right]
$$

in the momentum space, respectively. The functions $\rho\left(\mathbf{r}_{\mathbf{i}}\right)$ and $\gamma\left(\mathbf{p}_{\mathbf{i}}\right)$ are the one-electron densities and $\Gamma_{x}\left(\mathbf{r}_{1}, \mathbf{r}_{2}\right)$ and $\Pi_{x}\left(\mathbf{p}_{1}, \mathbf{p}_{2}\right)$ are the exchange densities in the position and momentum space respectively. Let us remark that, using Hartree-Fock functions, we are studying the Fermi correlation between same-spin electrons which arises from the antisymmetry of the wave function. In this sense, the term electron correlation, mentioned before, alludes to the statistical correlation. This point must be clarified in order to not lead to confusion if one considers a correlated system as one beyond the Hartree-Fock approximation (the Löwdin definition of correlation energy).

It should be noticed that the one-particle densities are marginal distributions of the twoparticle ones, because the former are obtained from the latter by integration, as $\rho\left(\mathbf{r}_{1}\right)=$ $\int \Gamma\left(\mathbf{r}_{1}, \mathbf{r}_{2}\right) d \mathbf{r}_{2}$ and $\gamma\left(\mathbf{p}_{1}\right)=\int \Pi\left(\mathbf{p}_{1}, \mathbf{p}_{2}\right) \mathrm{d} \mathbf{p}_{2}$. Thus, the information content of the marginals is not enough to determine the double-variable distribution they are coming from.

Different definitions of distance between two arbitrary distributions $f(\mathbf{x})$ and $g(\mathbf{x})$ appear, where both are defined over the same domain. The interest in finding appropriate tools for 'comparing' two different systems or processes in terms of distribution functions is justified by the strong connection between some of the above mentioned information measures and many relevant physical and chemical properties of those systems. The term 'distance' should be understood as a measure of how dissimilar the two functions are, not necessarily verifying all mathematical properties required for a true distance, as the usual quadratic distance does. However, all of them keep as main distance properties the non-negativity, the symmetry (invariance under exchange of functions) and saturation (minimal zero value only for identical distributions).

The relative entropy or Kullback-Leibler divergence (KL) [35] is one of the pioneering global measures of the difference between two arbitrary uni- or multivariate probability distributions. It expresses the amount of information supplied by the data for discriminating among the distributions, being a 'directed divergence' and therefore not symmetric:

$$
K L(f, g)=\int f(\mathbf{x}) \ln \frac{f(\mathbf{x})}{g(\mathbf{x})} \mathrm{d} \mathbf{x}
$$

Its applications for different procedures in obtaining minimum cross entropy estimations and the determination of atomic and molecular properties [29], among others, make it to constitute an essential tool within the information theory. Won and You introduced, somewhat implicity, a closely related information measure between two or more distributions, the Jensen-Shannon divergence (JSD) [51,52]:

$$
J S D(f, g)=\frac{1}{2}\left[K L\left(f, \frac{f+g}{2}\right)+K L\left(g, \frac{f+g}{2}\right)\right] .
$$

Consequently, JSD represents the mean dissimilarity (understood in terms of the KL measure) of each density respect to the mean one. 
This measure is strongly related to the Shannon entropy, which is defined for an arbitrary uni- or multivariate probability distribution as $S(f)=-\int \mathrm{d} \mathbf{x} f(\mathbf{x}) \ln f(\mathbf{x})$. Shannon entropy is well known to play a relevant role within an information-theoretic framework because it constitutes a measure of spreading of the density over its domain [53]. Attending to the JSD definition given above, the Jensen-Shannon divergence can be also expressed in terms of the Shannon entropy as

$$
J S D(f, g)=S\left(\frac{f+g}{2}\right)-\frac{1}{2}[S(f)+S(g)] .
$$

The JSD is characterized for quantifying the "Shannon entropy excess" of a couple of distributions with respect to the mixture of their respective entropies (mean density), defined in Eq. (7) as a particular case of the so-called weighted generalized divergences [54] with identical weights $1 / 2$. Apart from preserving the global character of the Shannon entropy, the JSD possesses the main properties required for a measure to be interpreted as an informational distance. In particular, its non-negativity is a consequence of the convexity of the $S(f)$ functional. Other important advantages of this divergence are that: (i) does not require the condition of absolute continuity for the probability distributions involved, (ii) weights of each density can be different from $1 / 2$ as appearing in Eq. (7), and (iii) can be generalized for an arbitrary number of distributions. During the past years researchers have been interested in work towards parametric generalizations of these classical measures of information. The JSD also admits other kind of generalizations, as will be shown elsewhere.

In this work we intend to perform an exhaustive analysis of atomic systems using JSD, on the basis of their two-electron densities, in the following two ways:

(i) Differences between the pair distribution $\Gamma\left(\mathbf{r}_{1}, \mathbf{r}_{2}\right)$ and the product of marginals $\rho\left(\mathbf{r}_{1}\right) \rho\left(\mathbf{r}_{2}\right)$ reveal the existence of some interconnection between its variables and quantify the effects from the exchange density on the systems under consideration. With JSD those differences are obtained for atomic systems (neutrals and ions), in both conjugated spaces (Sect. 3.1).

(ii) The ionization of a neutral system, by adding or removing an electron, modifies its structural characteristics. This was studied by means of the JSD between the one-particle distributions of the neutral and the ionized systems [55]. Here, a step further is taken, dealing with divergences among electron-pair densities, also in position and momentum spaces (Sect. 3.2).

\section{Numerical results}

Divergence measures allow us to quantify the differences between general systems, in particular by means of electron atomic densities. In this section we intend to employ JensenShannon divergence in order to analyse the particularities and characteristics of two-electron and one-electron densities. The Near-Hartree-Fock wavefunctions of Koga et al [56,57] are employed. Functions normalized to unity will be managed in what follows, for the sake of their interpretation as probability distributions.

\subsection{Interelectronic correlation analysis}

We will compute the Jensen-Shannon divergence between two-electron density and the product of one-electron densities, being one of the main reasons its property that its value is zero when the systems compared have no correlation. Thus, the Jensen-Shannon divergence 


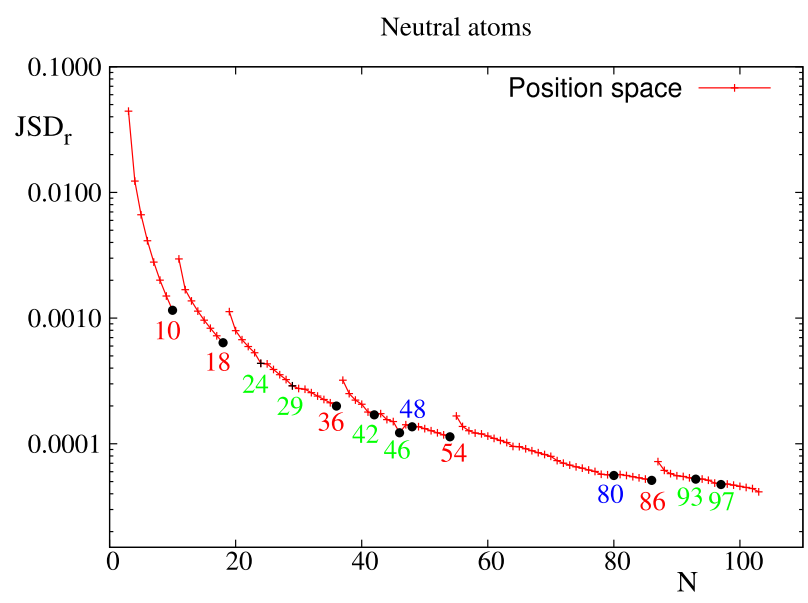

Fig. 1 Jensen-Shannon divergence in position space $\left(\mathrm{JSD}_{r}\right)$ between the electron pair density and the product of monoelectronic densities, for neutral atoms with number of electrons $N=3-103$. Labels are for systems with closed shells (red), closed subshells (blue) or anomalous shell-filling (green). Atomic units (a.u.) are used

acts as a measure of the disparity between two- and one-electron densities. These comparisons will be carried out on double variable distributions in both position $\left(\Gamma\left(\mathbf{r}_{1}, \mathbf{r}_{2}\right)\right.$ and $\rho\left(\mathbf{r}_{1}\right) \rho\left(\mathbf{r}_{2}\right)$ respectively) and momentum $\left(\Pi\left(\mathbf{p}_{1}, \mathbf{p}_{2}\right)\right.$ and $\gamma\left(\mathbf{p}_{1}\right) \gamma\left(\mathbf{p}_{2}\right)$ respectively) spaces. Let us remark that in this case, the last term in the JSD (see Eq. (7)) can be expressed as $-\left[\frac{S(\Gamma)}{2}+S(\rho)\right]$ in position space, and similarly for the momentum space. The systems under study are neutral atoms with a number of electrons $N \leq 103$ (nuclear charge $Z=N$ ), and singly-charged ions $(Z=N \pm 1)$ with $N \leq 54$.

Firstly, we computed the Jensen-Shannon divergence between the electron pair density and the product of the corresponding monoelectronic densities for neutral systems $N=2-103$ in position space. The obtained results are shown in Fig. 1. It can be observed the decreasing trend of JSD when the nuclear charge of the system increases, which indicates that the correlation decreases as the number of electrons increases. This was an expected result, as the number of electrons is known to determine how strong or weak is the relevance of the correlation between the mentioned electrons. Thus, in the case that we have information about the position of an electron within a group of three, this knowledge becomes much more relevant than if we have a group of thirty electrons, when the information about just one does not tell us much more about the other twenty nine.

Notwithstanding, some additional patterns are noticeable, most certainly related to the shell filling structure. This fact was emphasized in a previous work [58] for systems within the shorter range $N \leq 36$, on the basis of the mutual information as quantified by the $K L$ divergence among distributions, as here done by means of JSD up to $N=103$. The trend is monotonous, without many exceptions for a long period, however some discontinuities appear when changing from a period to another indicating a transition to a new electronic shell. Each segment can be assigned to a particular shell, in the fashion the figure shows. Besides, some less relevant extrema appear, all of them related to anomalous shell filling systems. Some specific systems have been labelled, as follows:

- Closed shells: $N=10,18,36,54,86$. Thus noble gases appear as minima systematically. The same was observed in the $K L$ divergence for systems up to $N \leq 36$ [58]. 
- (i) Anomalous shell-filling: minima $N=42$, 46 (outer d-subshell), with valence subshell half-filled and filled, respectively. (ii) The same applies to $N=24,29$ : in spite of not being extrema, the curve displays a 'change of slope' at those points, to be contrasted later with momentum space. (iii) Minor extrema appear for heavy systems, within a region of anomalies.

- Closed subshells: minima $N=48,80$ with filled d-subshell.

The Jensen-Shannon divergence between electron pair density and the product of monoelectronic densities in momentum space hardly provides additional information. The conclusions we arrive are similar than before, in fact, the curves in both spaces almost overlap each other, as it can be seen by comparing Fig. 2 (momentum space) with the previous one (note the identical scales of both figures). Thus the electron pair densities in momentum space are providing roughly the same information as the position ones.

Nevertheless, additional remarks are in order. Comparing curves in position and momentum space, it is observed that (i) the aforementioned 'changes of slope' at $N=24,29$ in position space are displayed as maxima in momentum space; (ii) previous minimum $N=42$ translates now to $N=43$, also with outer d-subshell; (iii) instead of minimum $N=80$ it is now observed the maximum $N=78$. No extrema appear in the heavy systems region.

Notice that just mentioned systems $N=24,29,42,43,46,78$ have a non-filled inner ssubshell and an outermost d-subshell (the inner one is empty for the system $N=46$, clearly highlighted in both figures).

As observed in Ref. [58] for KL, most JSD values are higher in position than in momentum space. The opposite applies for a few systems with anomalous shell-filling. Particularly relevant are the cases $N=24,29$ with half-filled $4 s$ inner orbital and outermost $3 d$ subshell (half-filled and completely filled, respectively). Their $\mathrm{JSD}_{p}$ (Fig. 2) are greater than the respective $\mathrm{JSD}_{r}$ (Fig. 1) by $17 \%$ and $25 \%$. Such amount belongs to the range $8-13 \%$ for heavier systems with similar anomalies: $N=41,42,44,45,47$ (subshells $5 s 4 d$ ), and $N=$ 78,79 (subshells $6 s 5 d$ ), all them with an inner half-filled $s$-orbital. It is concluded that, for most systems, correlation effects are weaker in momentum space, the opposite being true for the aforementioned anomalous systems, in a slight fashion with few exceptions. These results support and extend the conclusions obtained in Ref. [58] for systems up to $N=36$, regarding the relative behaviors in position and momentum spaces.

The above discussion was focused on neutral atoms and their properties, but in what follows we will consider also some of their corresponding ions. Let us now use the JensenShannon divergence, JSD, in order to quantify the differences between the electron distributions at the one- and two-electron levels, now for ionized systems. In doing so, Fig. 3 shows the JSD between the monoelectronic and the electron pair density of different ions, calculated in both conjugated spaces. First let us focus on Fig. 3a where singly-charged cations with number of electrons $N=2-54$ have been taken into account. The curves show us a decreasing behaviour when $N$ increases and, in this case, the momentum space roughly presents smaller values of JSD. However, an interesting pattern appears in the figure: cations corresponding to $N=10,18,23,28,36,41,46,48,54$ have a minor difference, a lower value of JSD, in both conjugated spaces with the only exception $N=48$ (extremely light minimum, only in position space). Among maxima (located at almost identical positions in both spaces), let us emphasize the presence of $N=24,29,42$. Regarding these two collections, some comments are in order:

- As for neutral atoms, noble gases $N=10,18,36,54$ are displayed as lower peaks. On the other hand, alkalines $N=3,11,19,37$ appear as maxima. Similarly occurs, in position 


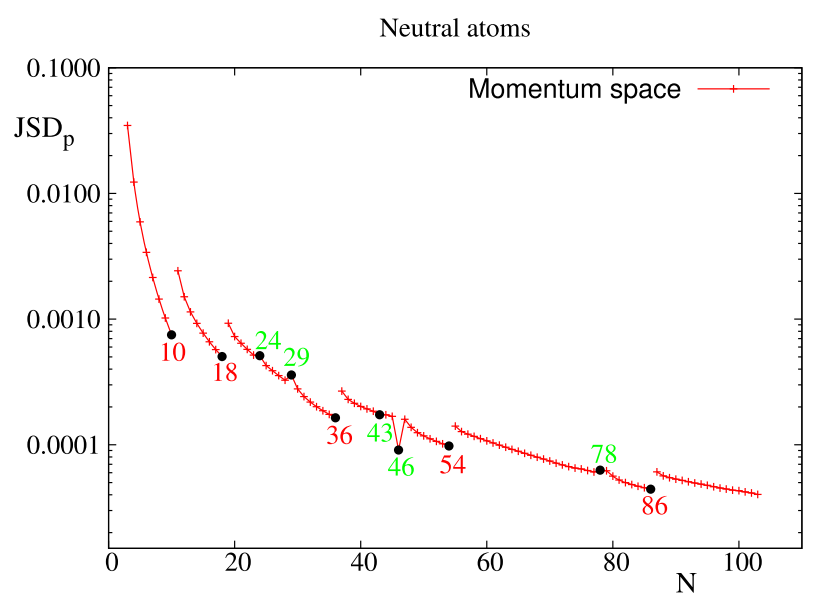

Fig. 2 Jensen-Shannon divergence in momentum space $\left(\mathrm{JSD}_{p}\right)$ between the electron pair density and the product of monoelectronic densities, for neutral atoms with number of electrons $N=3-103$. Labels are for systems with closed shells (red) or anomalous shell-filling (green). Atomic units (a.u.) are used

space, with the closed-shell system $N=48$ (minimum) and its neighbors $N=47,49$ (maxima).

- Pairs of extrema 23-24 and 28-29 (minimum-maximum) are due to the anomalous shell filling of both maxima $N=24,29$, with the inner half-filled $4 s$ subshell, and with half-filled or filled (respectively) outermost $3 d$ subshell.

- Similarly the pair of consecutive extrema 41 - 42 has inner half-filled $5 s$ subshell, and outermost subshell $4 d$, half-filled for the maximum $N=42$.

- Particularly interesting is the strong minimum $N=46$, as discussed for neutrals, with empty $5 s$ subshell and filled valence subshell $(4 d)$.

- A few of the above systems display a higher JSD in momentum than in position space: $N=24,29,42,47$ (with inner half-filled s-orbital and outermost half-filled or completely filled d-orbital), as well as $N=25$ (half-filled outermost d-orbital).

In Fig. 3b, where the anions have been considered, exactly the same pattern is observed, but with a less elaborate structure, due to the fewer available systems as it was previously mentioned. Some alkaline earths have been labelled, but let us emphasize the 'absence from the list' of alkalines, which are displayed as maxima for cations. Position space JSD is almost systematically greater than the momentum one. The only extremely light exceptions are $N=41-42$, with differences between spaces below $0.2 \%$.

\subsection{Atomic ionization}

Applying divergence measures to the analysis of ions will allow us to find which electrons have a higher impact on the whole density. In order to do that, we inspect the Jensen-Shannon divergence between the pair densities of an ion (cation or anion) and its respective neutral, in both position and momentum spaces. For completeness, similar results on the basis of one-particle densities, previously obtained [55], are also displayed. This is shown in Figs. 4 and 5.

Next results are to be interpreted as measures of the 'amount of change' on the electron cloud of a given system after its ionization takes place, either by ejecting or by capturing an 


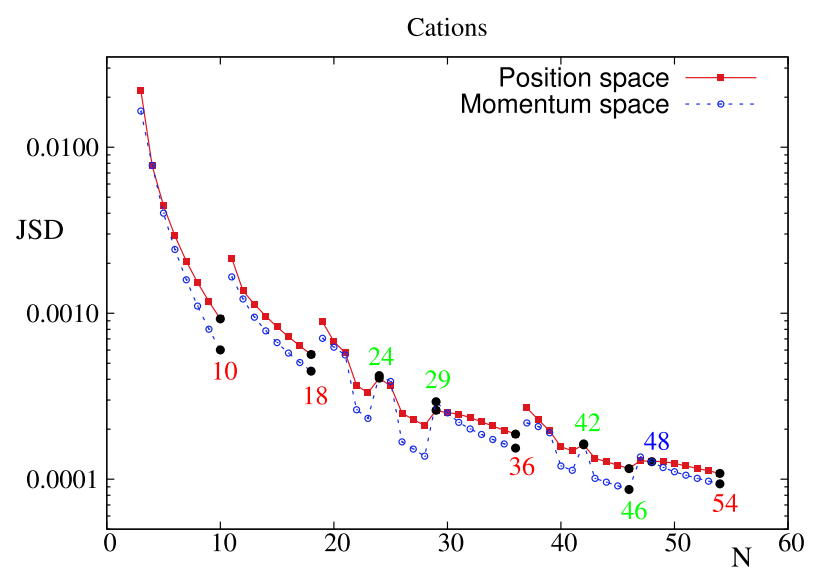

(a) Cation comparison

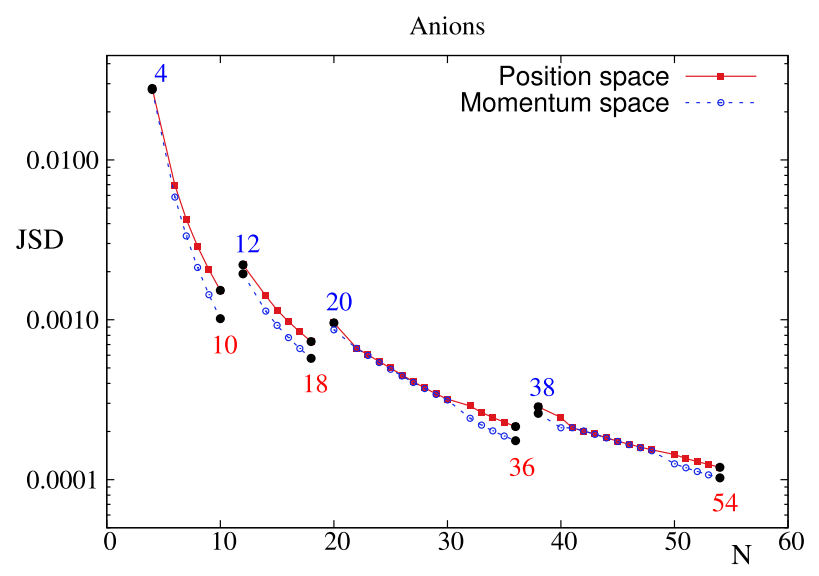

(b) Anion comparison

Fig. 3 Jensen-Shannon divergence, in position and momentum spaces, between the electron pair density and the product of one-particle densities, for $\mathbf{a}$ cations and $\mathbf{b}$ anions with number of electrons $3 \leq N \leq 55$. Labels are for systems with closed shells (red), closed subshells (blue) or anomalous shell-filling (green). Atomic units (a.u.) are used

electron. Thus, if the initial neutral system has $N$ electrons, the resulting ion will have $N-1$ or $N+1$ electrons, for the cation and the anion, respectively. This interpretation applies to both conjugated spaces, as well as to one-particle and electron pair densities.

Figure 4 encloses curves of JSD between the electron distribution of a neutral system and that of its singly-charged cation, denoted in position and momentum spaces as $\operatorname{JSD}_{r}(N, C)$ in Fig. 4a, and $\operatorname{JSD}_{p}(N, C)$ in Fig. 4b, respectively. A simple function of the atomic ionization potential AIP needed for performing such process is also depicted. This function has been considered in order to better compare all curves, which display a variety of local extrema, most of them characterized accordingly with the shell-filling of neutrals and ions, as discussed below. 


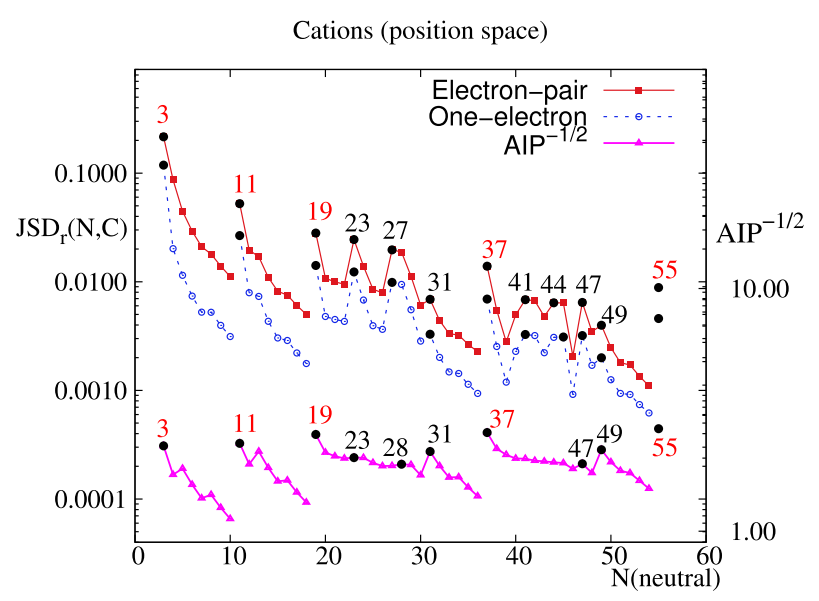

(a) Cation comparison in $\mathrm{r}$ space

Cations (momentum space)

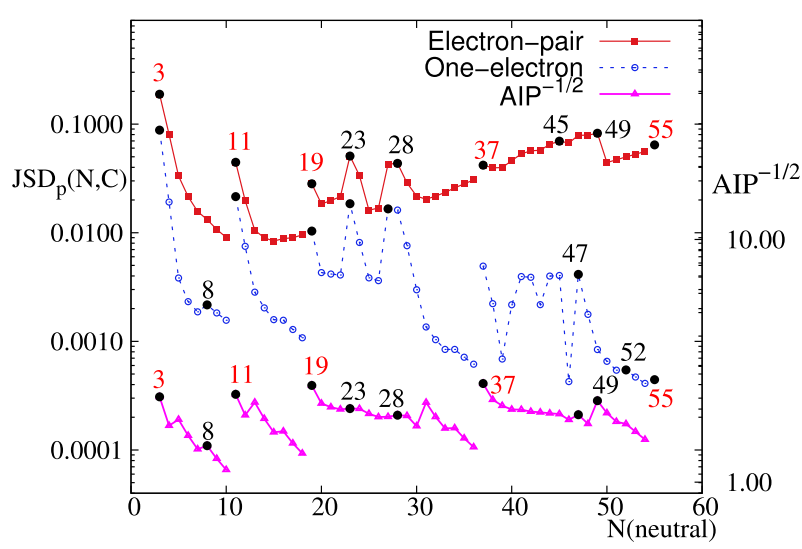

(b) Cation comparison in p space

Fig. 4 Pair and one-electron Jensen-Shannon divergences between the neutral system with a number of electrons $3 \leq N \leq 55$ and its singly charged cation, and the inverse of square root of the atomic ionization potential (AIP), in a position and $\mathbf{b}$ momentum spaces. Atomic units (a.u.) are used

Let us start with the location of maxima of JSD in position space, when comparing a neutral of $N$ electrons with the resulting cation of $N-1$ electrons, namely $\operatorname{JSD}_{r}(N, C)$, as displayed in Fig. 4a. In the two-particle case, higher peaks are found at neutrals $N=3,11,19,23,27,31,37,41,44,47,49,55$. Within the range of $N$ here considered, it is worthy to remark:

- Alkalines $N=3,11,19,37,55$ appear included, that is systems which outermost ' $s$ ' subshell becomes empty after ionization.

- The same applies to the anomalous $N=47$ regarding its valence subshell $5 \mathrm{~s}$.

- For systems $N=31,49$ the lost subshell is $4 p$ and $5 p$, respectively.

- Rest of peaks $(N=23,27,41,44)$ correspond to ' $d$ ' valence subshells. 
- Comparing with the one-electron curve, almost identical structures are obtained regarding their extrema and monotonic trends. The most prominent differential feature is the systematic higher value of electron pair JSD as compared to the monoelectronic one.

The same analysis has been carried out in momentum space, and the results are displayed in Fig. 4b. Conclusions are similar to those obtained from position-space divergences, and the differences are emphasized below:

- Position-space peaks $N=3,11,19,23,37,55$ (enclosing all alkalines) remain in momentum space for both one- and two-particle comparisons. On the other hand, $N=31$ is lost also in both cases.

- $N=27,45$ are kept in the monoparticular case, while the slight shifts from maxima located at $(27,44)$ to $(28,45)$ are observed for electron pair divergences.

- Maximum $N=49$ disappears for momentum one-electron distributions, while $N=$ 41,47 for the two-electron ones. Notice that the half-filled 5 s subshell of systems $N=41$ and $N=47$ becomes filled and empty, respectively, after ionization.

- On the other hand, the disappearance of $N=31,49$ from the set of maxima in going from position to momentum space corresponds to systems which ' $p$ ' valence subshell becomes empty. Instead, the divergence at the one-electron level displays maxima $N=8,52$, characterized for getting a half-filled ' $p$ ' valence subshell after ionization.

- Concerning asymptotic trends, it is observed that the monoelectronic JSD remains within a low interval for large $N$, while the electron pair divergence appreciably increases, roughly from $N=25$ on.

The differences between the JSD behaviors in position and momentum spaces arise from the asymptotic behaviors of the involved densities. This fact has been shown in previous studies on density functional analysis, particularly JSD among others [41]. In position space, the atomic one- and two-electron densities have an exponential decreasing behavior, so that the values of the involved integrals are mainly determined by the regions surrounding the nuclei, those almost unaffected by ionization. However, the lowspeed regions are associated with the outermost subshells, being consequently those mainly determining the JSD values. The differences between the valence subshells of the neutral and ionized systems are more clearly reflected in the pair distribution, as compared to the monoelectronic one.

Let us analyse the structure of the JSD in each period comparing their extremal values to those of AI P. In Table 1, values of number of electrons $N$ of the neutral system for which the Jensen-Shannon divergence display local extrema (maximum) are given. In addition, minimum values of the $A I P$ are shown, which are associated to systems with a single electron in the valence subshell, making consequently such a subshell to disappear after ionization, and the resulting system to strongly differ from the initial one. This relevant difference is usually revealed in terms of a high divergence and dissimilarity between the initial and the final system. There exist a strong correlation also with the structure displayed by the atomic ionization potential $A I P$ in the $N C$ ionization process in which an 's' electron is removed, as shown in the corresponding column of the table. However, as it can be observed the same is not true when the removed electron is of 'p' or ' $d$ ' type.

It appears natural to wonder ourselves about all the above features when the initial neutral system gets an additional electron, so becoming a singly-charged anion. The appropriate calculations have been performed, and the results obtained are displayed in Fig. 5.

Conclusions for anions are much more limited, as compared to those obtained for cations. The reason is the absence of numerous systems from the list of anions, not included in the Ref. [56] considered for the present work. However, some interesting remarks are in order. 
Table 1 Number of electrons $N$ at local extrema for the atomic ionization potential AIP of neutral atoms and the Jensen-Shannon divergences in position $\left(\mathrm{JSD}_{r}\right)$ and momentum $\left(\mathrm{JSD}_{p}\right.$ ) spaces, between the electron pair densities of a neutral system with a number of electrons $3 \leq N \leq 54$ and its singly charged anions $(A)$ and cations $(C)$. Atomic units are used

\begin{tabular}{llll}
\hline Measure & & $N \longrightarrow C$ & $A \longrightarrow N$ \\
\hline AIP & $(\mathrm{s})$ & $3,11,19,23,28,37,47,55$ & \\
& $(\mathrm{p}, \mathrm{d})$ & $5,8,13,16,31,34,49$ & \\
$\mathrm{JSD}_{r}$ & $(\mathrm{~s})$ & $3,11,19,23,27,37,41,44,47,55$ & $3,11,19,24,29,37,42,44,46$ \\
& $(\mathrm{p}, \mathrm{d})$ & 31,49 & \\
$\mathrm{JSD}_{p}$ & $(\mathrm{~s})$ & $3,11,19,23,28,37,45,55$ & $3,11,19,24,29,46$ \\
& $(\mathrm{p}, \mathrm{d})$ & 49 & $15,49,53$ \\
\hline
\end{tabular}

In position space, Fig. 5a, maxima for the divergence neutral-anion are roughly the same as those of neutral-cation, for the one- and the two-electron distributions. In both cases, the following patterns are displayed:

- Alkalines $N=3,11,19,37$ remain as maxima, also for anions. Let us remark that the next alkaline $(N=55)$ is not included in the managed anion set.

- The anion obtained from neutral $N=31$ does not give rise to a maximum, contrary to the cation.

- Two sets of slight shifts are observed: (23, 27 and 41) to (24, 29 and 42), and (44 or 45, and 47$)$ to (44 and 46).

The only exception to the above common patterns is $N=49$, shifted to $N=51$ in the monoelectronic case, and lost for electron pair densities.

In momentum space, Fig. 5b, (i) alkalines $N=3,11,19$ are kept for anions, (ii) the oneelectron shifts of maxima $(8,23,27$ or $28,41,45,47,52)$ to $(7,24,29,42,44,46,51)$ include the two-electron ones, corresponding to $N=24,29,46$, and (iii) in both cases, the new maximum $N=15$ appears, as well as $N=33$ at the one-electron level, that is systems with a half-filled ' $p$ ' valence subshell, as also $N=51$ included in the above list of shifts.

Differences between the global behaviors of the one- and two-electron curves appear justified in the same way as for neutral-cation transitions. That is, both densities decrease exponentially far from the nucleus in position space, while the asymptotic fall of momentum densities is much more slow.

\section{Conclusions and future work}

Jensen-Shannon divergence (JSD) has been employed as comparative functional for different many-electron atoms on the basis of their respective one- and two-electron distributions, enclosing neutral atoms $(N=Z)$ and singly-charged ions $(N=Z \pm 1)$. Particularly JSD is applied to quantify the interelectronic correlation in atomic systems. A thorough study was carried out, by considering (i) both position and momentum conjugated spaces, and (ii) systems with a nuclear charge as far as $Z=103$.

The correlation among electrons was measured by comparing, for an arbitrary system, the double-variable electron pair density with the product of the respective one-particle densities. As expected, the absence of correlation translates into the extreme allowed value JSD $=0$, 


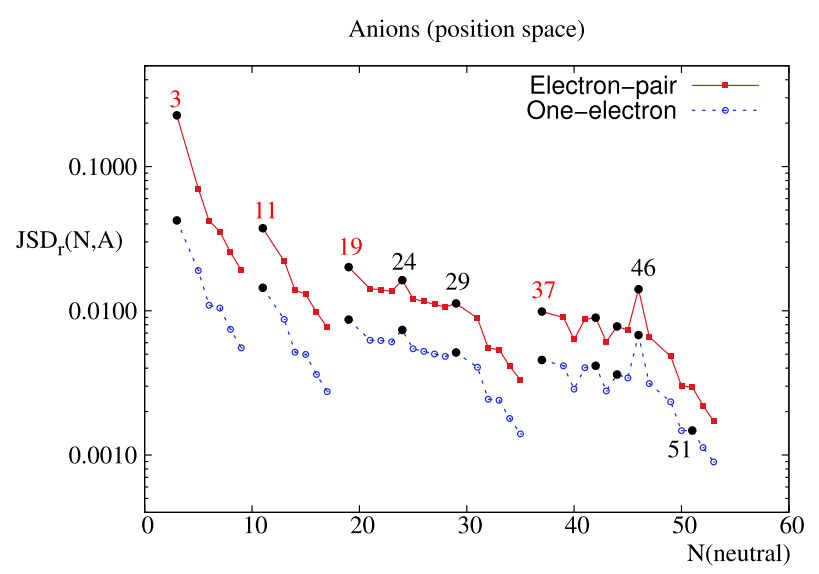

(a) Anion comparison in $\mathrm{r}$ space

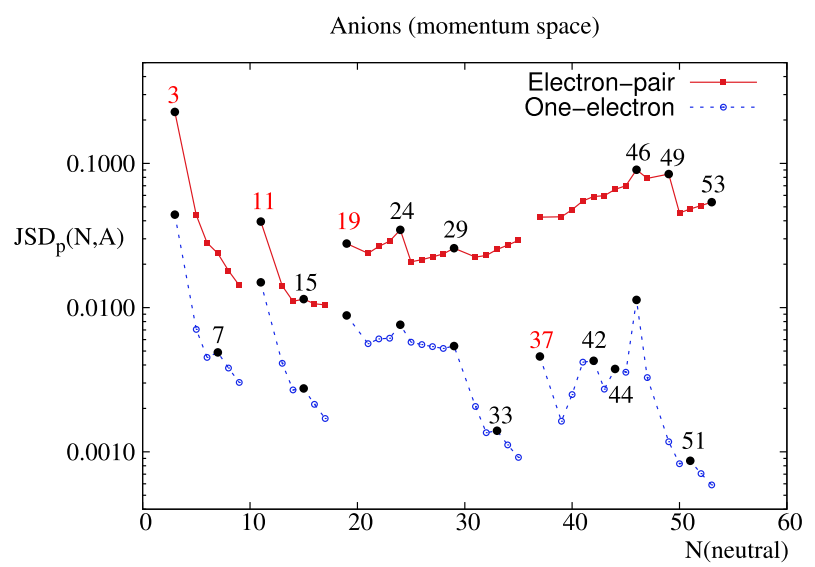

(b) Anion comparison in $\mathrm{p}$ space

Fig. 5 Pair and one-electron Jensen-Shannon divergences between the neutral system with a number of electrons $3 \leq N \leq 54$ and its singly charged anion, in a position and $\mathbf{b}$ momentum spaces. Atomic units (a.u.) are used

and any deviation from the above value is caused by the existence of correlation, more or less strong, accordingly with the relevance of the aforementioned deviations.

The main conclusion is that the dependence on the number of electrons only governs general trends of JSD, being insufficient as single variable to justify the structural patterns shown by those curves. Interelectronic correlation roughly decreases as the number of electrons increases, as revealed by the decreasing behavior of JSD in terms of $N$. However, there appear a number of local extrema, whose location and strength are clearly determined by shell-filling patterns. Specially relevant changes in JSD are observed just after adding an electron to a closed-subshell system. All this is valid in position and momentum spaces.

The above comments on neutral atoms apply also for cations and anions, regarding structural patterns. Once again the interelectronic correlation, as measured by JSD, decreases along a given period (i.e. as adding electrons) but notably increases in passing to a new period. 
Apart from correlation, additional properties of atomic systems were analysed by means of JSD. Such is the case of ionization as described by the 'resemblance' among neutrals and their respective singly-charged ions, in terms of the corresponding electron pair densities. While structural patterns are similar in position and momentum spaces, the same is not true regarding monotonic behaviors. JSD in both spaces displays a decreasing trend for light systems, that remains in the position case for the whole set of systems. However, it turns out to an increasing one in momentum space for medium-heavy systems. So break of resemblance after ionization appears greater in momentum space than in the position one, for both cations and anions.

Future work is planned by considering other functionals. For instance, relative Fisher information (containing also the gradient of the density) was exhaustively applied for the analysis of specific one-particle quantum-mechanical systems (Morse potential and harmonic oscillator [48], hydrogen-like atoms [49]). Additionally, numerous studies of atomic [59] and molecular [60] systems were performed by means of the quantum similarity index on the basis of their one-particle densities. On the other hand, a study of different divergences and similarity measures, particularly Jensen-Shannon divergence, between the exchange density and the product of the one particular densities could be interesting.

Acknowledgements This work was supported in part by the Spanish MINECO project FIS2014-59311-P (cofinanced by FEDER). A.L.M., J.C.A. and J.A. belong to the Andalusian research group FQM-020, and S.L.R. to FQM-239.

Funding Open Access funding provided thanks to the CRUE-CSIC agreement with Springer Nature.

Open Access This article is licensed under a Creative Commons Attribution 4.0 International License, which permits use, sharing, adaptation, distribution and reproduction in any medium or format, as long as you give appropriate credit to the original author(s) and the source, provide a link to the Creative Commons licence, and indicate if changes were made. The images or other third party material in this article are included in the article's Creative Commons licence, unless indicated otherwise in a credit line to the material. If material is not included in the article's Creative Commons licence and your intended use is not permitted by statutory regulation or exceeds the permitted use, you will need to obtain permission directly from the copyright holder. To view a copy of this licence, visit http://creativecommons.org/licenses/by/4.0/.

\section{References}

1. T. Koga, Electron-Pair Densities of atom. in Many-Electron Densities and Reduced Density Matrices (Springer, New York, 2000)

2. M. Rodríguez-Mayorga, M. Via-Nadal, M. Solà, J.M. Ugalde, X. Lopez, E. Matito, Electron-pair distribution in chemical bond formation. J. Phys. Chem. A 122, 1916-1923 (2018)

3. R.P. Sagar, N.L. Guevara, Mutual information and correlation measures in atomic systems. J. Chem. Phys. 123, 044108 (2005)

4. J. Cioslowski, Topology of Electron Correlation. in Many-Electron Densities and Reduced Density Matrices (Springer, New York, 2000)

5. P.W. Ayers, E.R. Davidson, Necessary conditions for the n-representability of pair distribution functions. Int. J. Quant. Chem. 1006, 1487-1498 (2006)

6. M. Levy, P. Ziesche, The pair density functional of the kinetic energy and its simple scaling property. J. Chem. Phys. 115, 9110-9112 (2001)

7. Á. Nagy, Density-matrix functional theory. Phys. Rev. A 66, 022505 (2002)

8. D.A. Mazziotti, Two-electron reduced density matrix as the basic variable in many-electron quantum chemistry and physics. Chem. Rev. 112, 244-262 (2012)

9. M. Higuchi, K. Higuchi, Pair density functional theory. Comput. Theor. Chem. 1003, 91-96 (2013)

10. M. Higuchi, K. Higuchi, A pair density functional theory utilizing the correlated wave function. J. Phys. Conf. Ser. 150, 042056 (2009)

11. P. Ziesche, Attempts toward a pair density functional theory. Int. J. Quantum Chem. 60, 1361-1374 (1996) 
12. G. Li Manni, R.K. Carlson, S. Luo, D. Ma, J. Olsen, D.G. Truhlar, L. Gagliardi, Multiconfiguration pair-density functional theory. J. Chem. Theory Comput. 10, 3669-3680 (2014)

13. J.L. Bao, Y. Wang, X. He, L. Gagliardi, D.G. Truhlar, Multiconfiguration pair-density functional theory is free from delocalization error. J. Phys. Chem. Lett. 8, 5616-5620 (2017)

14. M. Sajjan, K. Head-Marsden, D.A. Mazziotti, Entangling and disentangling many-electron quantum systems with an electric field. Phys. Rev. A 97, 062502 (2018)

15. R. Benesch, V.H. Smith, Correlation and X-ray scattering. I. Density matrix formulation. Acta Cryst. 26, 579-586 (1970)

16. A.J. Thakkar, A.N. Tripathi, V.H. Smith, Molecular X-ray- and electron-scattering intensities. Phys. Rev. A 29, 1108-1113 (1984)

17. T. Koga, Y. Kasa, A.J. Thakkar, Accurate algebraic densities and intracules for heliumlike ions. Int. J. Quantum Chem. 46, 689 (1993)

18. J.M. Herbert, J.E. Harriman, Comparison of two-electron densities reconstructed from one-electron density matrices. Int. J. Quantum Chem. 90, 355 (2002)

19. C. Sarasola, J.M. Ugalde, R.J. Boyd, The evaluation of extracule and intracule densities in the first-row hydrides, $\mathrm{LiH}, \mathrm{BeH}, \mathrm{BH}, \mathrm{CH}, \mathrm{NH}, \mathrm{OH}$ and $\mathrm{FH}$, from self-consistent field molecular orbital wavefunctions. J. Phys. B: At. Mol. Opt. 23, 1095 (1990)

20. J. Cioslowski, B.B. Stefanov, A. Tan, C.J. Umrigar, Electron intracule densities with correct electron coalescence cusps from Hiller-Sucher-Feinberg-type identities. J. Chem. Phys. 103, 6093 (1995)

21. A.J. Proud, D.E.C.K. Mackenzie, J.K. Pearson, Exploring electron pair behaviour in chemical bonds using the extracule density. Phys. Chem. Chem. Phys. 17, 20194-20204 (2015)

22. C.E. Shannon, W. Weaver, The Mathematical Theory of Communication (University of Illinois Press, Urbana, 1949)

23. R.A. Fisher, Theory of statistical estimation. Proc. Cambridge Phil. Soc., 22, 700-725, Reprinted in Collected Papers of R.A. Fisher, edited by J.H. Bennet (University of Adelaide Press, South Australia) 1972, 15-40 (1925)

24. J. Pipek, I. Varga, Universal classification scheme for the spatial-localization properties of one-particle states in finite, d-dimensional systems. Phys. Rev. A 46, 3148 (1992)

25. R. López-Ruiz, H.L. Mancini, X. Calbet, A statistical measure of complexity. Phys. Lett. A 209, 321-326 (1995)

26. B.R. Frieden, Science from Fisher Information (Cambridge University Press, Cambridge, 2004)

27. T.M. Cover, J.A. Thomas, Elements of Information Theory (Wiley, New York, 1991)

28. S.R. Gadre, R.D. Bendale, S.P. Gejji, Analysis of atomic electron momentum densities: use of information entropies in coordinate and momentum space. Chem. Phys. Lett. 117, 138 (1985)

29. M. Ho, V.H. Smith, D.F. Weaver, C. Gatti, R.P. Sagar, R.O. Esquivel, Molecular similarity based on information entropies and distances. J. Chem. Phys. 108, 5469 (1998)

30. K.D. Sen, C.P. Panos, K.C. Chatzisavvas, C.C. Moustakidis, Net Fisher information measure versus ionization potential and dipole polarizability in atoms. Phys. Lett. A 364, 286-290 (2007)

31. S. Janssens, A. Borgoo, C. Van Alsenoy, P. Geerlings, Information theoretical study of chirality: enantiomers with one and two asymmetric centra. J. Phys. Chem. A 112, 10560-10569 (2008)

32. R.F. Nalewajski, Information principles in the theory of electronic structure. Chem. Phys. Lett. 372, 28 (2003)

33. J.C. Angulo, J. Antolín, Atomic complexity measures in position and momentum spaces. J. Chem. Phys. 128, 164109 (2008)

34. J.C. Angulo, J. Antolín, K.D. Sen, Fisher-Shannon plane and statistical complexity of atoms. Phys. Lett. A 372, 670-674 (2008)

35. S. Kullback, A. Leibler, On information and sufficiency. Ann. Math. Stat. 22, 79-86 (1951)

36. J.H. Lin, Divergence measures based on the Shannon entropy. IEEE Trans. Inf. Theory 37, 145-151 (1991)

37. B. Fuglede, F. Topsoe, Jensen-Shannon divergence and Hilbert space embedding. IEEE Int. Sym. Inf. Theo. 31, 71-73 (2004)

38. P.W. Lamberti, A.P. Majtey, A. Borras, M. Casas, A. Plastino, Metric character of the quantum JensenShannon divergence. Phys. Rev. A 77, 052311 (2008)

39. I. Grosse, P. Bernaola-Galván, P. Carpena, R. Román-Roldán, J.L. Oliver, H.E. Stanley, Analysis of symbolic sequences using the Jensen-Shannon divergence. Phys. Rev. E 65, 041905 (2002)

40. J.C. Angulo, J. Antolín, S. López-Rosa, R.O. Esquivel, Jensen-Shannon divergence in conjugate spaces: the entropy excess of atomic systems and sets with respect to their constituents. Phys. A 389, 899 (2010)

41. J. Antolín, J.C. Angulo, S. López-Rosa, Fisher and Jensen-Shannon divergences: quantitative comparisons among distributions. Application to position and momentum atomic densities. J. Chem. Phys. 130, 074110 (2009) 
42. J.C. Angulo, S. López-Rosa, J. Antolín, Generalized Jensen divergence analysis of atomic electron densities in conjugated spaces. Int. J. Quantum Chem. 111, 297-306 (2011)

43. A.L. Martín, S. López-Rosa, J.C. Angulo, J. Antolín, Jensen-Shannon and Kullback-Leibler divergences as quantifiers of relativistic effects in neutral atoms. Chem. Phys. Lett. 75, 642 (2015)

44. J. Antolín, J.C. Angulo, S. Mulas, S. López-Rosa, Relativistic global and local divergences in hydrogenic systems: a study in position and momentum spaces. Phys. Rev. A 90, 042511 (2014)

45. S.J.C. Salazar, H.G. Laguna, R.P. Sagar, Higher-order statistical correlations in three-particle quantum systems with harmonic interactions. Phys. Rev. A 101, 042105 (2020)

46. S.J.C. Salazar, H.G. Laguna, R.P. Sagar, Higher-order information measures from cumulative densities in continuous variable quantum systems. Quantum Rep. 2, 560-578 (2020)

47. O. Werba, A. Raeber, K. Head-Marsden, D.A. Mazziotti, Signature of van der Waals interactions in the cumulant density matrix. Phys. Chem. Chem. Phys. 21, 23900 (2019)

48. T. Yamano, Relative fisher information for Morse potential and isotropic quantum oscillators. J. Phys. Commun. 2, 085018 (2018)

49. T. Yamano, Relative fisher information of hydrogen-like atoms. Chem. Phys. Lett. 691, 196-198 (2018)

50. P.O. Löwdin, Quantum theory of many-particle systems. I. Physical interpretations by means of density matrices, natural spin-orbitals, and convergence problems in the method of configurational interaction. Phys. Rev. 97, 1474 (1955)

51. A.K.C. Wong, M. You, Entropy and distance of random graphs with application to structural patternrecognition. IEEE Trans. Pattern Anal. Mach. Intell. 7, 599 (1985)

52. C.R. Rao, T. Nayak, Cross entropy, dissimilarity measures, and characterizations of quadratic entropy. IEEE Trans. Inf. Theory 31, 589 (1985)

53. C.E. Shannon, A mathematical theory of communication. Bell Syst. Tech. J. 27, 379 (1948)

54. L.E. Riveaud, D. Mateos, S. Zozor, P.W. Lamberti, Generalized divergences from generalized entropies. Phys. A 68-76, 510 (2018)

55. S. López-Rosa, J. Antolín, J.C. Angulo, R.O. Esquivel, Divergence analysis of atomic ionization processes and isoelectronic series. Phys. Rev. A 80(1-9), 012505 (2009)

56. T. Koga, K. Kanayama, S. Watanabe, A.J. Thakkar, Analytical Hartree-Fock wave functions subject to cusp and asymptotic constraints: He to Xe, Li+ to Cs+, H- to I-. Int. J. Quantum Chem. 71, 491 (1999)

57. T. Koga, K. Kanayama, T. Watanabe, T. Imai, A.J. Thakkar, Analytical Hartree-Fock wave functions for the atoms Cs to Lr. Theor. Chem. Acc. 104, 411 (2000)

58. R.P. Sagar, H.G. Laguna, N.L. Guevara, Electron pair density information measures in atomic systems. Int. J. Quantum Chem. 111, 3497 (2011)

59. J.C. Angulo, J. Antolín, Atomic quantum similarity indices in position and momentum spaces. J. Phys. Chem. 126, 044106 (2007)

60. R. Carbó-Dorca, X. Girones, P.G. Mezey (eds.), Fundamentals of Molecular Similarity (Kluwer Academic, Dordrecht, 2001) 